REDUCTION OF SLITLESS SPECTRA -

THE DETECTION OF FAINT EMISSION LINES

H.-M. Adorf, H.-J. R\&ser

Max-Planck-Institut fur Astronomie, Heidelberg

\title{
ABSTRACT
}

We have developed a spectra reduction system producing relative spectrophotometry directly from prism as well as from grating spectra plates. Lines and other spectral features are detected, and their essential parameters are measured automatically. A deep quasar survey using this technique is now underway.

\section{MOTIVATION AND AIMS}

This work was motivated by the questions: "Why are so few quasars known with redshifts greater than 3.5 ? Are they overlooked by the present survey techniques?" The usual spectral survey method has been "to look at the plates" in order to detect obvious emission lines. Although the human eye definitely is an excellent "image processor", it does not work quantitatively (and fails on low contrast images). It is therefore difficult to attack cosmological problems such as the determination of the quasar space density. These problems require a well defined sample of quasars, crucially depending on the survey's completeness.

We decided to investigate the advantages of quantitative survey work, which should result in a homogeneously selected sample with a definite completeness limit and should make different quantitative surveys comparable with each other. Spectrophotometry from the survey plate is also required, because follow-up observations with big telescopes for large numbers of faint objects would cost too much time to be feasible.

An ideal reduction procedure should maximize both completeness of the sample and the reliability of the findings. It should (1) certainly be automated, (2) be "self-adaptive", $i$. e., work effectively very close to the noise, and (3) quickly and reliably single out the spectra with the most "unusual" features. The data together with the procedures used should define what is unusual. Specifically, the procedure should determine the limiting equivalent width of a line as a function of the wavelength, the continuum magnitude, and the line width. 
The search for high-redshift quasars requires deep plates in the red. In order to test our spectra reduction system (SRS) we used a blue plate instead, which contains several known quasars and quasar candidates. The plate was taken at the $80 / 120 / 240 \mathrm{~cm}$ Schmidt telescope on Calar Alto (Spain) in 1981; and kindly loaned to us by Dr. Haug (Hamburg), who is pursuing his own visual survey for emission line objects. The plate measures $24 \mathrm{~cm} \times 24 \mathrm{~cm}$ and covers a projected area of $5.7 \mathrm{deg} \times$ $5.7 \mathrm{deg}$. The unwidened spectra, dispersed by the "thin" 1.7 deg objective prism, were recorded on a baked Kodak IIIa-J emulsion. Between 350 and $520 \mathrm{~nm}$ the reciprocal linear dispersion rises from 75 to $245 \mathrm{~nm} / \mathrm{mm}$, with $139 \mathrm{~nm} / \mathrm{mm}$ at $\mathrm{H}_{\gamma}$. Thus, the resolving power under $2^{\prime \prime}$ seeing declines from 200 down to 90 , with 135 at $\mathrm{H}_{\gamma}$. In 75 minutes a sky background of about $22.7 \mathrm{mag} / \mathrm{arcsec} 2$ produces a plate density of 1 , and a background signalto-noise ratio of 20 in an area of 1 arcsec 2 .

\section{FIRST PHASE IN REDUCTION: FROM RAW DATA TO SPECTRAL INTENSITIES}

In establishing the limits of a survey technique we are concerned with the properties of a whole sample of spectra. In order to avoid preselecting this set, we scanned sub-areas (or frames) of the plate instead of single spectra using our PDS-microdensitometer with an aperture of $10 \mu \mathrm{m} \mathrm{x} 10 \mu \mathrm{m}$ and a step size of $5 \mu \mathrm{m}$. Thus one frame, measuring $2000 \mathrm{x}$ 2000 pixels, covers less than 2 permille of the total Schmidt plate area, or roughly $1 / 20 \mathrm{deg} 2$. Presently, each frame is then graphically displayed, and "usable" spectra, which surpass a 2 (background) threshold above the local background, are interactively selected. A frame contains 30 to 40 of such usable spectra. The limiting brightness of the plate exceeds $20 \mathrm{mag}$.

All usable spectra are then reduced with procedures comparable to those used by others (Clowes et al. 1980, Christian 1982, Vaucher et al. 1982), which result in a "one-dimensional, background-subtracted, noisesuppressed, wavelength-calibrated spectral intensity trace" along each of the usable two-dimensional spectra. As an example, Figure 1 shows the intensity trace of the quasar PHL 938, which one frame was centered on. Details concerning the SRS can be found elsewhere (Adorf 1984). Note that in the present work we used a constant contrast $\gamma=3.84$ for density to intensity conversion, and did not correct for the varying spectral sensitivity of the emulsion. Also, the wavelength scale is preliminary only and rather uncertain in the green.

\section{SECOND PHASE IN REDUCTION: THE DETECTION OF LINES AND OTHER FEATURES}

Since we are trying to detect quasars with arbitrary redshifts by the presence of emission lines, we must be prepared to detect a line of unknown (but presumably broad) shape, of unknown intensity, at an unknown position on an unknown (but presumably flat), noisy continuum. In order 
to detect a line, measure its position and derive a confidence parameter we cross-correlate a given spectrum with a set of (Gaussian) template profiles differing only in their full width at half maximum (FWHM). The correlation peak determines the detected line's FWHM and also its similarity to the template. (The detection of features other than lines, such as continuum breaks, proceeds similarly, and only requires a different set of template profiles.) Next the spectrum is convolved with an appropriate linear filter to measure the line flux or its equivalent width.

Distinguishing noise bumps from true lines remains a problem: Ideally, the frequency distribution of a measured quantity, such as the "similarity" or the equivalent width, in the whole sample of spectra, should tell us which lines are probably caused by statistical fluctuations, and how to choose the right detection threshold. As an example, Figure 2 shows a scatter diagram of the measured line equivalent widths versus the J-magnitude of the object to which it belongs, for a preselected line FWHM of $10 \mathrm{~nm}$. From the observed frequency distribution of equivalent widths we read a current lower limit of around $12 \mathrm{~nm}$ for the detection of such a line in a spectrum of an object brighter than 18 th mag.

The ability of our SRS to select a complete sample of spectra has been tested against three colleagues who, using a microscope, visually examined 200 spectra within a test area on a different, red plate. Their task was to search for emission or absorption lines, and to assign a "degree of certainty" to their findings. The visual surveyors seemed to differentiate best on moderately dense spectra, but worked inefficiently at the faint and the bright ends, as has also been reported by others (Haug 1983). At the important faint end, all of the visually detected emissionline-candidate spectra were also found by the SRS (which found $50 \%$ more spectra in all). The ability of the reduction system to reject artifacts with a definite signature (e. $g$. dust and spectrum overlaps), however, remains to be improved.

\section{CONCLUSION}

We have developed an automated spectra reduction system (SRS), which produces spectrophotometry directly from slitless spectra plates. The SRS detects and measures spectral features such as lines, and has proven not only to be complete in its findings but also to go deeper than visual surveyors. The ability of the SRS to discriminate against dust and other faults, however, remains to be improved. The SRS is currently used for a quantitative deep spectral quasar-survey on plates with photographically recorded prism spectra, and on films with electronographically recorded grating spectra. Our SRS is general enough so that other applications could be considered as well. 


\section{REFERENCES}

Adorf, H.-M. 1984: Ph. D. thesis (in preparation)

Christian, C. A. 1982: Astrophys. J. Suppl. Ser. 49, pp. $555-592$

Clowes, R. G., Emerson, D., Smith, M. G., Wallace, P. T., Cannon, R. D., Savage, A., Boksenberg, A. 1980: Mon. Not. R. Astr. Soc. 193, pp. $415-426$

Haug, U. 1983: private communication

Vaucher, B., Kreid1, T. J., Thomas, N. G., Hoag, A. A. 1982: Astrophys. J. 261 , pp. $18-24$

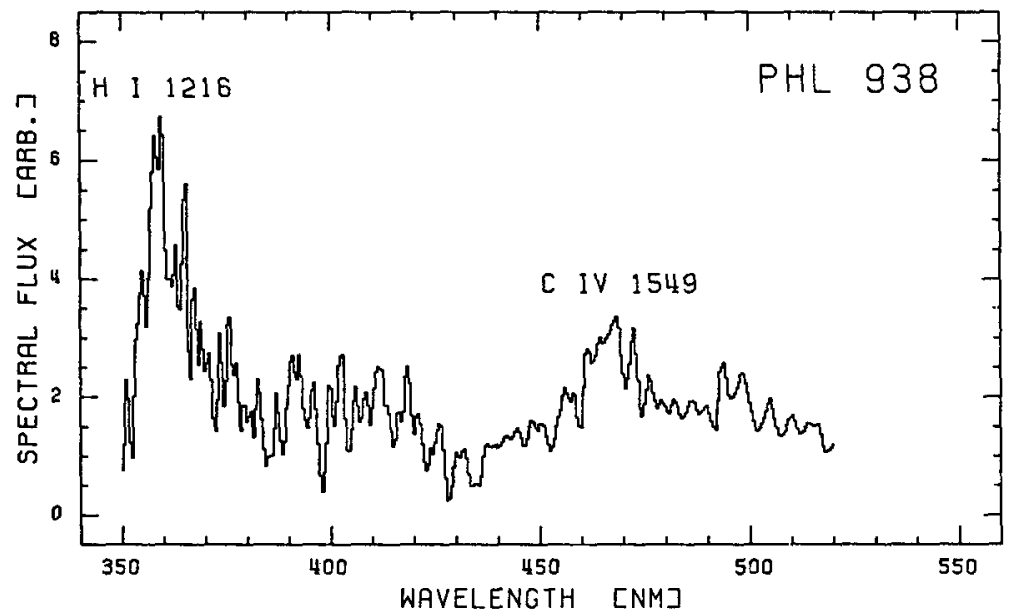

Figure 1: Relative spectral flux of the quasar PHL $938(B=17.49, z=$ 1.955) as derived by the spectral reduction system.

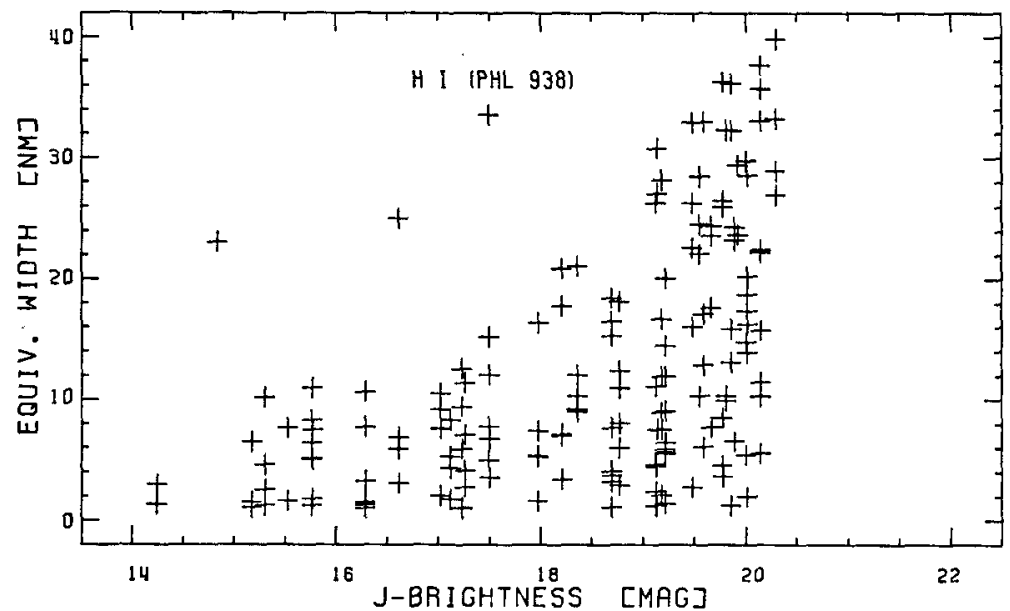

Figure 2: Scatter diagram of measured equivalent widths versus the Jmagnitude of the object to which the line (candidate) belongs. 